\title{
Internet-based framework to support integration of the customer in the design of customizable products
}

\author{
J A Ninan* and Z Siddique \\ School of Aerospace and Mechanical Engineering, University of Oklahoma, Norman, Oklahoma, USA \\ The manuscript was received on 31 March 2006 and was accepted after revision for publication on 4 September 2006.
}

DOI: 10.1243/09544054JEM599

\begin{abstract}
Integration of customers is a necessary element to design and produce customer centric products. Design tools and methodologies need to be altered to accommodate customers into the process of designing customized products. In the current paper a mass customization framework is presented, that uses computer-aided design (CAD) and finiteelement-based optimization tools to integrate the customer into the design process via the internet. A mass customization template for generating optimized user-customized products is also presented. The capability of the system is demonstrated by a case study on customization of bicycle frames.
\end{abstract}

Keywords: mass customization, customer centric product development

\section{INTRODUCTION}

Researchers have categorized mass customization (MC) in several different manners based on different factors. Gilmore and Pine [1] identify four basic approaches to provide customization: collaborative, adaptive, cosmetic, and transparent. Managers can combine these approaches successfully to provide MC. Duray and Milligan [2] categorized MC based on the point of external customer involvement in design, fabrication, assembly, or delivery stages of the manufacturing process for the product. Another widely used taxonomy, based on production management, separates products into four categories relative to the different intersections of customer orders with the entire production process, also called the customer order decoupling point (CODP) [3]. The four categories of products are: made-to-stock (MTS); assemble-to-order (ATO); made-to-order (MTO); and engineer-to-order (ETO).

Integration of the customer in the design process is required to support $\mathrm{MC}$, which involves providing ETO or MTO products. The internet can be used to

*Corresponding author: School of Aerospace and Mechanical Engineering, University of Oklahoma, Norman, Oklahoma, USA.email: jijuandrews@ou.edu

Paper originally presented at the 12th ISPE International Conference on Concurrent Engineering: Research and Applications, Dallas, USA, 2005. communicate and integrate the customer in a real time with the manufacturer. During ETO or MTO, questions that need to be answered are: is the configuration specified by the customer feasible and are the customer-specified parameters for the configuration optimum? Although constraints and rules on how different options can be combined to allow configuration change and bounds on input parameters can reduce the number of infeasible configurations, this does not ensure that a feasible product will be generated from customer specifications. Moreover, a user-specified design needs to be optimized, subject to design and manufacturing constraints. Having a design analyst analyse each specified configuration is not an efficient solution to the problem and will require a considerable amount of resources to support such a system. Consequently, to achieve customization of products in real time, the product design modelling and evaluation tools need to be automated and enhanced to enable operation over the internet. In the context of MC variety management, the modelling is different from traditional product modelling in that product data have to be related to both the specific product variant and the common platform that supports the range of product variety.

Simpson et al. [4] developed a web-based customization system for refiner plates for pulp and paper processing. Flores et al. [5] presented a similar web-based system for customizing coated steel belt 
Table 1 Categorization of modularity, scaling and attributes for product family information architecture

Modularity

Platform modules: The core functions and structure for all customized varieties.

Optional modules: Added/deleted to the platform to provide functional varieties. The optional modules are

divided into three sub-categories.

(1) User selected modules: either directly selected by the customer or directly based on optional functions selected by the customer.

(2) Dependent modules: not directly related but dependent on the modules/options selected by the customer.

(3) Performance modules: based on performance and scaling parameters specified by the customer.

Scaling

Size parameters: Customize the product size

Performance and constraint parameters: these user inputs cannot be used directly to scale the product; instead analysis and optimization of the configuration with user input are required to satisfy these performance and constraint parameters.

Attributes

Specify the attributes such as material, coating, finishing operation etc.

sheaves based on parametric modifications. Zha and Lu [6] developed a web-based knowledge system to support product family design for a power supply product family. Saxon and Beaulieu [7] developed a web-based engineering system, which can automatically perform finite element analysis (FEA) of stabilizer bars.

Siddique and Zhou [8] presented a template-based approach to represent the product family. Siddique and Boddu [9] presented an agent-based framework automatically to generate three-dimensional (3D) models of customer-specified designs. A product family computer-aided design (PF-CAD) module was developed to implement the design templates and automatically generate solid models of family members associated with the product platform. Siddique and Ninan [10] extended the PF-CAD template to accommodate the automated FEA of product families for internet-based architecture to automate FEA of family members. The research presented in the current paper extends this work.

In section 2 the web-based framework, template and optimization formulation are presented. In section 3, the capability of the developed framework is demonstrated through customization of bicycle frames. Concluding remarks are presented in section 4

\section{WEB-BASED FRAMEWORK TO SUPPORT ETO}

To support ETO customization, the PF architecture information can be categorized into modularity, scaling, and attributes. Modularity is used to specify the architecture of the PF. The architecture defines not only the hierarchy of the product, but also relationships among modules and components. Modules can have children, with the lowest level consisting of components. Scaling is used to stretch or shrink one or more variables of the platform and options to satisfy customer requirements. During MC both configuration and scaling changes have to be supported. In many instances the customization of products also depends on attributes. These attributes might be used for external physical appearance, such as colour, decals, etc or might affect the performance and feasibility of the product (materials, coating, finishing operation etc.). Attributes can be selected by the user or determined by analysis. Table 1 shows the categorization of modularity, scaling, and attributes.

The framework presented posseses the capability automatically to build and analyse each product variant specified by the customer. Moreover, userspecified design needs to be optimized, subject to design and manufacturing constraints. The framework collects user selections and specifications regarding the customization of the product using a web-based interface. These parameters and selections are then used automatically to build the CAD model of the product variety. This is done with the help of a MC CAD/FEA template that generalizes the approach of building the model, analysing, and optimization of user customizable products. The customer-specified configuration is optimized for weight and performance.

The framework is implemented in the Microsoft windows operating system utilizing internet information system (IIS), Microsoft.NET Framework, and ANSYS FEA and optimization tools. The implementation consists of two servers. Server I is the web server that holds the ASP.NET-based web user interface for the customization of products. Server II is the computer aided engineering (CAE) server that holds ANSYS finite element and optimization tools.

ANSYS is automated to perform the task normally done through the user interface using its application programmable interface (API) scripting language called APDL. APDL macros have .mac extension and can be edited using a text editor. For the 


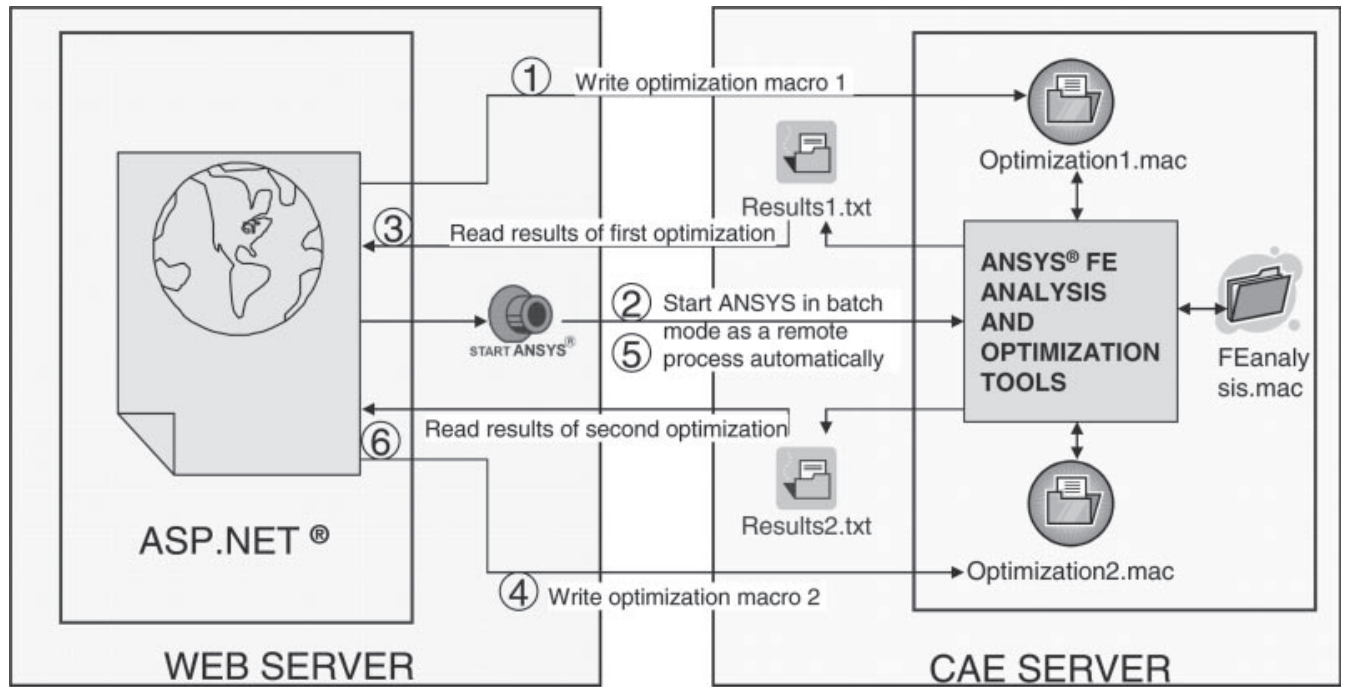

Fig. 1 Activities in the MC process

set-up, two macros have been developed for optimization, each corresponding to the optimization formulations presented in section 2.2. ASP.NET writes these macros using the user inputs and selections. The FEA macro is the APDL implementation of the CAD/FEA template for automatically analysing the products, which includes building the geometry, applying loads and boundary conditions, solving and querying the required results automatically. The macro first generates the feasible configuration from the user-specified functions and then scales the model to appropriate size. The generated model is parameterized to allow optimization to be performed on the model. The sequences of steps involved in the customization process are shown Fig. 1. Steps 4-6 are optional and are only required if a second optimization step is necessary. Communication between ASP.NET and ANSYS is performed using text files as both ANSYS and ASP have the capability to open and read text files. Invoking ANSYS is performed as a Windows process using the capability of Microsoft. net.

\subsection{Combined MC CAD and FEA template}

The MC CAD/FEA template is a set of well-defined and structured guidelines and instructions that generalizes the geometry construction and analysis information for all product varieties (Table 2). The template uses a top-down approach to define the product varieties using the platform as the core. The different varieties, $P_{i}$, can be generated by the addition of the product platform with optional modules. For the purpose of generating the CAD and the FEA model, information that needs to be included in the template are: assembly, geometry, material, mesh, and loading information. Certain assembly and loading information is contained in the product family level, whereas some are contained in the module $\left(\mathrm{ppm}_{j}\right.$ and $\left.\mathrm{pom}_{s}\right)$ and component $\left(C_{\mathrm{pp}}\right.$ and $C_{\mathrm{po}}$ ) level respectively. The elements of $P_{i}$ include PP, PO, AssemCon, and LoadCond. This means a product variety should embrace the product platform (PP), utilize product options (PO) to provide the varieties, assembly constraints (AssemCon) to specify spatial relationships among them. The loading conditions (LoadCond) and boundary conditions (BoundaryCond) are also specified to complete the structural model for FEA. In the template, it is assumed that (PP) is not null or an empty set. To provide varieties the (PO) set cannot be empty either. PO is a set of modules that provide the same functionality. As for PP and PO, they could be a single component or a module. Inside the PP or POs, there are components and component-related characteristics, which include geometric, hierarchy, and parametric information. At the component level they also hold the information for the building the finite element model. This information includes material properties, element type, element sizes, boundary conditions, loads, and loading types associated with each component.

\subsection{Optimization formulation}

A two-step process, as shown in Fig. 1, is utilized to optimize the model of the customized product. The first step is to optimize the customized product with the user-specified configuration and parameters used as constants. The general optimization formulation for the first step is as follows.

The optimization formulation for this step is given by

Find: Values of $X$

Subject To:

$g_{j}(X) \leqslant k$ 
$f^{\wp}$

$L_{x_{\mathrm{u}}} \leqslant x_{\mathrm{u}} \leqslant U_{x_{\mathrm{u}}}$

Minimize: $\sum_{i=l}^{m} \Phi_{i}(X)$

Where: $\sum_{i=l}^{m} \Phi_{i}(X)$ is the aggregate objective function

$X_{\mathrm{u}}\left(x_{\mathrm{u}} \in \stackrel{i=l}{X_{\mathrm{u}}}\right)$ is the set of design variables not related to the user specifications
$X_{\mathrm{r}} \cup X_{\mathrm{u}}=X$

$g_{j}=$ are the side constraints (performance, stress, etc.) $j$ varies from 1 to $\mathrm{m}$

$f^{\wp}$ relates the user specifications with $X_{\mathrm{r}}$.

In the first step there are two types of design variables: (1) variables, $X_{\mathrm{r}}$, that are dependent on the user inputs and (2) variables, $X_{\mathrm{u}}$, that are

Table 2 Structure of CAD/FEA template to support mass customization

$$
\begin{aligned}
& P F=\{P P, P O, \text { AssemCon, LoadCon }\} \\
& P P=\left\{p p m_{1}, p^{\prime} m_{2}, \ldots ., p^{\prime} m_{j}\right\} \\
& P O=\left\{\left(p o_{1}^{1}, p o_{2}^{1}, \ldots \ldots, p o_{a}^{1}\right),\left(p o_{1}^{2}, p o_{2}^{2}, \ldots \ldots, p o_{b}^{2}\right), \ldots \ldots .\left(p o_{1}^{x}, p o_{2}^{x}, \ldots \ldots, p o_{z}^{x}\right)\right\}
\end{aligned}
$$

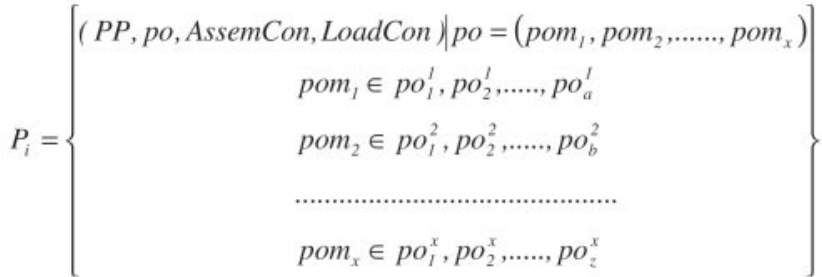

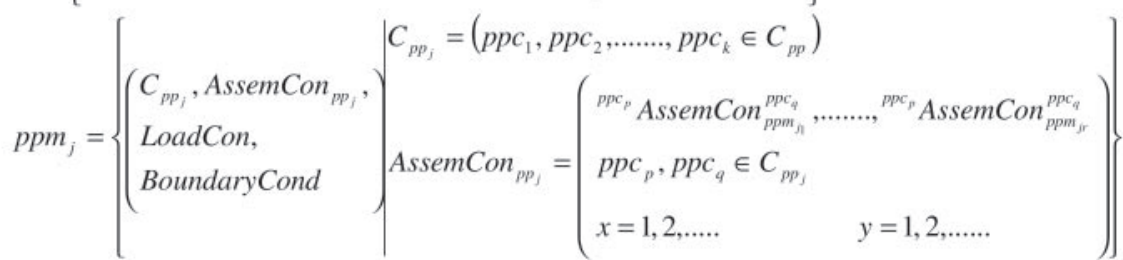

$$
\begin{aligned}
& p p c=(\text { Geom }, \text { Material }, \text { ElementType, ElementSize, LoadCon }, \text { BoundaryCond })
\end{aligned}
$$

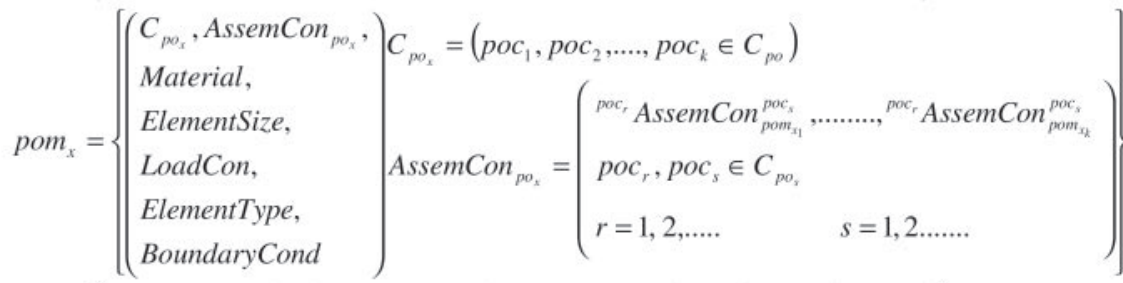

$$
\begin{aligned}
& \text { poc }=(\text { Geom }, \text { Material }, \text { ElementType, ElementSize, LoadCond }, \text { BoundaryCond }) \\
& \text { BoundaryCond }=\text { set of boundary conditions for the component. Each } \\
& \text { boundary condition contains location, degrees of freedom, and Magnitude } \\
& \text { AssemCon }=\left\{\begin{array}{l}
m_{v_{v}} \text { Assem Con }{ }_{1}^{m_{1}}, \ldots \ldots \ldots . . .{ }^{m_{x}} \text { Assem } \text { Con }_{z}^{m_{y}} \\
m_{v}, m_{w}, m_{x}, m_{y} \in P P \cup P O
\end{array}\right\} \\
& { }^{x} \text { AssemCon }_{i}^{y}=\left\{\text { Assem }_{M}, \text { Assem }_{R}\right\} \\
& P P \neq\{\varnothing\}, \quad \text { and } \quad P O \neq\{\varnothing\}
\end{aligned}
$$

\section{where}

PF $=$ Product Family

$P_{i}=$ Customized Product Variety

$P P=$ Product Platform

$\mathrm{ppm}_{j}=$ Product Platform Modules

$P O=$ Product Options

pom $_{s}=$ Product Option Modules
$C_{p p}=$ product platform component set

$C_{p o}=$ product option component set

${ }^{x}$ AssemCon ${ }^{y}=$ Assembly Constraint between

element $x$ and element $y$

Assem $_{M}=$ Assembly Method

Assem $_{R}=$ Assembly Reference 


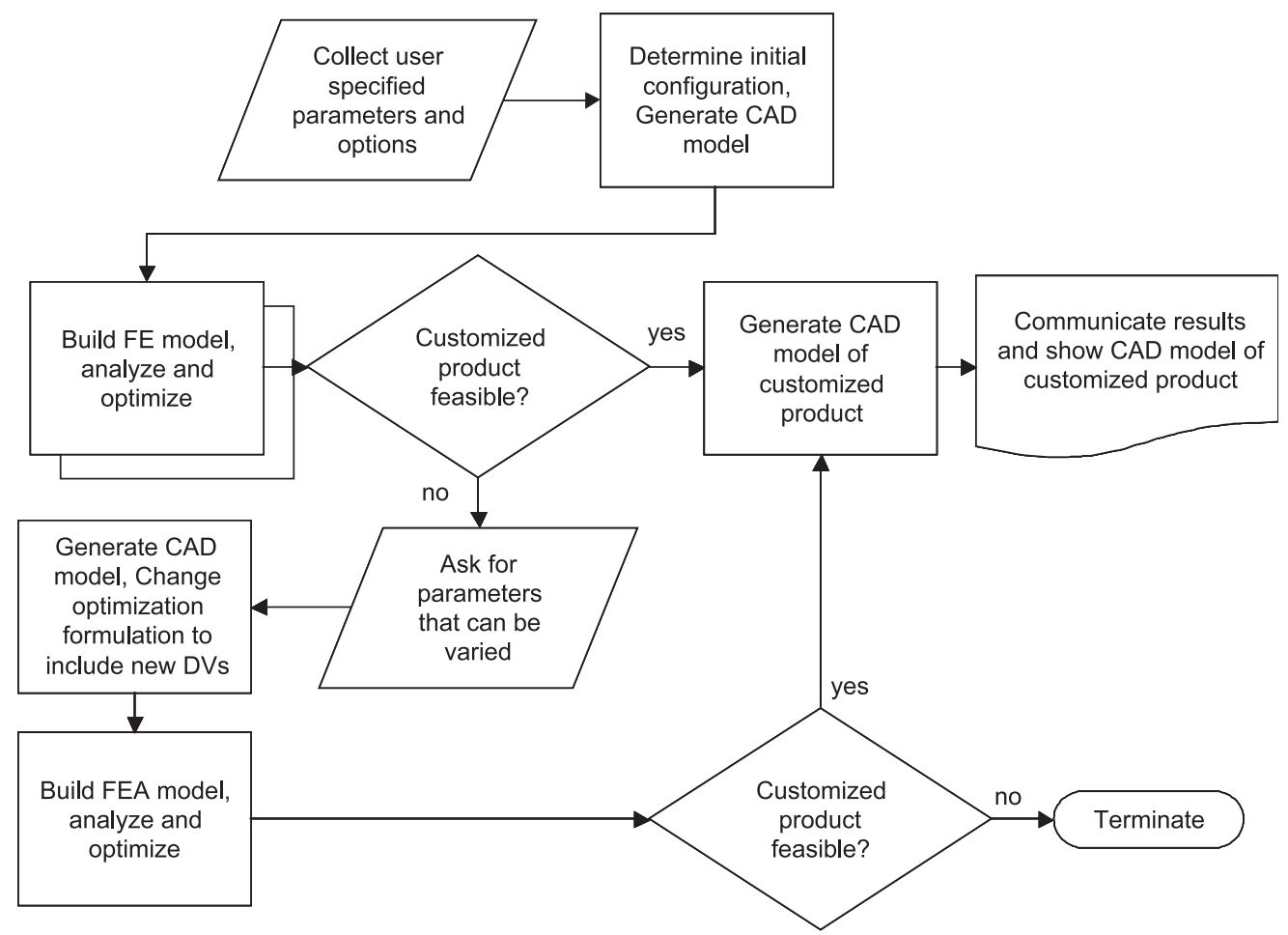

Fig. 2 Implementation

independent of the user input. The dependence of $X_{\mathrm{r}}$ on the user inputs is treated as a constraint in the problem formulation. The solution of the problem tries to find the values of $X_{\mathrm{u}}$ that will optimize the customized product.

If the optimization procedure does not yield a feasible design the user is asked to select the dimensions that can be relaxed. Optimization is then performed using the selected dimensions as design variables that vary \pm 10 per cent of initial value. In case of a feasible design CAD model of the configuration, geometric dimensions and specifications of the customized design are shown to the customer as feedback. The general formulation for this step is

$$
\begin{aligned}
& \text { Find: Values of } X \\
& \text { Minimize: } \sum_{i=l}^{m} \Phi_{i}(X) \\
& \text { Subject To: } g_{j}(X) \leqslant k \\
& f^{{ }^{\prime}} \\
& 0.9 \times f_{\mathrm{var}}^{\wp} \leqslant x_{r} \leqslant 1.1 \times f_{\mathrm{var}}^{\wp} \\
& L_{x_{\mathrm{u}}} \leqslant x_{\mathrm{u}} \leqslant U_{x_{\mathrm{u}}}
\end{aligned}
$$

where $X_{\mathrm{r}}=X_{\mathrm{r}}^{\prime} \cup X_{\mathrm{r}}^{\mathrm{var}}$ with $f^{\wp \prime}$ relating user input with parameters $\left(X_{\mathrm{r}}^{\prime}\right)$ that are not flexible, and $f_{\mathrm{var}}^{\wp}$ relating user input with set of parameters $\left(X_{\mathrm{r}}^{\mathrm{var}} ; x_{\mathrm{r}}^{\mathrm{var}} \in X_{\mathrm{r}}^{\mathrm{var}} ; f^{\wp \prime} \cup f_{\mathrm{var}}^{\wp}=f^{\wp}\right)$ that are flexible and can be varied. The rest of the variables are as presented in the step 1 formulation. The solution to the second step will result in values of $X_{\mathrm{r}}^{\mathrm{var} \prime}$ and $X_{\mathrm{u}}$ for the given objective function.

\section{CASE STUDY - CUSTOMIZABLE BICYCLE FRAMES}

\subsection{Bicycle product family}

A rider's performance, endurance, and comfort on a bicycle are a direct function of how well the bicycle fits the rider's proportions [11]. The MC framework presented in section 2 is implemented to offer customized bicycle frames. Three models are available for customization: men's, women's, and tandem. Customization of frames is a four-step process. The first step involves selecting the model to customize. In the second step (Fig. 3) the user is prompted to enter information regarding the dimensions of the intended design and also the rider's weight. The user is prompted for the values of the following parameters: (a) the weight of the rider; (b) desired arm reach (seat to handlebar); (c) total length of the frame; (d) total height of the frame; (e) floor to seat height; (f) wheel radius; and (g) clearance. These parameters are then passed to the finite element software from the web page. FEA and optimization of the user configuration is carried out in real time. If the user specifications lead to an infeasible configuration then the user is prompted to select the dimension that can be varied. A second iteration is carried out including the selected dimensions as well as the design variables (DVs), so that a feasible configuration can be derived at. 


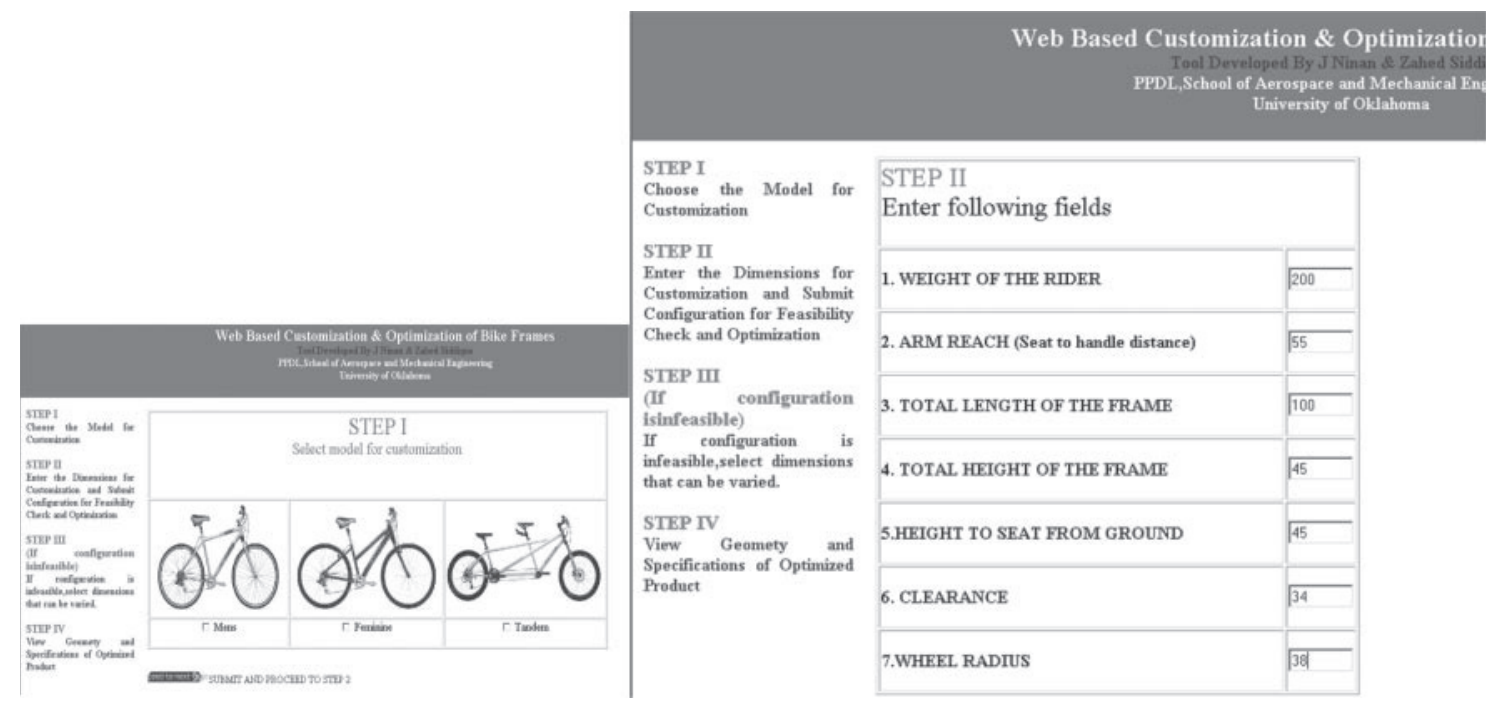

Fig. 3 Web-based user interface for customization showing steps 1 and 2

The newly added DVs are allowed to vary within a range of +10 per cent of their initial value. In case of a feasible design, the dimensions of the resulting model and the geometry are shown to the user as a feedback. Optional analysis information can also be included in the HTML result file generated by ANSYS. Currently the system only allows two iterations. If the second optimization run (step III in Fig. 3) leads to an infeasible configuration, the system communicates the result and then prompts the user to change the geometry and exits of the loop. The application of the MC-CAD/FEA template presented in section 2.1 is shown in Table 3.

ST, CSTL, CSTR, CSBL, and CSBR are the product platform components. TL, LTM, LTT, LTTF, HTTL, HTTM, HTTT, HTTF, STF, DTT HTLS, HTLM, HTLL, HTMS, HTMM, HTML, HTTS, HTTM, HTTL form the optional components for the family. Figure 4 shows the various geometry parameters for the products. AssemRel relates the geometry and spatial relations among the various components. The LoadConds and Boundconds (Fig. 5) describe the loading and boundary conditions for the finite element formulation. Finite element modelling and material information for each component are also contained in the template.

The MC-CAD/FEA template also contains information regarding the type of elements to be used and the relation between different geometric entities, the material properties, and the application of loads and constraints for different product family members. The template provides the guidelines and the knowledge base for generalizing the analysis and optimization of the entire family. Aluminium T- 6 is assumed as the material for the frames. The template developed will vary from product to product in terms of parameters and other product specific data, but the type of information is generic and is addressed in the general template. The framework is general and can be used for customization of different products.

\subsection{User customized variety}

The implemented bicycle frame MC framework is demonstrated by customizing a bicycle frame through the developed user interfaces. First the men's model was selected and then values of parameters were specified to customize and design the frame. The values for the input parameters $\left(A_{\wp}\right)$ specified by the user are: (a) weight of the rider = $100 \mathrm{Kg}$; (b) arm reach $=56 \mathrm{~cm}$; (c) total length of frame $=98 \mathrm{~cm}$; (d) total height of frame $=84 \mathrm{~cm}$; (e) height to seat $=84 \mathrm{~cm}$; (f) clearance $=34 \mathrm{~mm}$; and $(\mathrm{g})$ wheel radius $=38 \mathrm{~cm}$. The optimization formulation for first iteration is as follows.

\section{First optimization formulation}

Find: Values of

$$
\begin{aligned}
& X_{\mathrm{r}}=(\mathrm{C}, \mathrm{D}, \mathrm{E}, \mathrm{J}, \mathrm{G}, \mathrm{H}) \\
& X_{\mathrm{u}}=(\mathrm{A}, \mathrm{B}, \mathrm{F}) \\
& f^{\varsigma}
\end{aligned}
$$

Minimize: Mass of the customized frame

Subject to: von Mises stress in the members $\leqslant$ $82.7 \times 10^{6} \mathrm{~Pa}$

Design variables:

$$
\begin{aligned}
& 72^{\circ} \leqslant \mathrm{A} \leqslant 75^{\circ}, 70^{\circ} \leqslant \mathrm{B} \leqslant 74^{\circ}, 3^{\circ} \leqslant \mathrm{F} \leqslant 4^{\circ} \\
& \mathrm{HT}=\text { head tube module HT } \\
& \mathrm{HT}=\left\{\mathrm{HTM}_{\mathrm{S}}, \mathrm{HTM}_{\mathrm{M}}, \mathrm{HTM}_{\mathrm{L}}\right\}
\end{aligned}
$$

In the first step $X_{\mathrm{r}}$ consists of the design variables that are dependent on the user inputs and (2) $X_{\mathrm{u}}$ Variables $\{A, B, F\}$ that are not dependent on the user inputs (Fig. 5). The initial iteration failed to result in a feasible product. On the second iteration the parameters height to seat (HS) and arm reach 
(AR) were selected as the dimensions that can be varied. Hence the optimization formulation for this specific case

\section{Second optimization formulation}

Find: Values of

$$
\begin{aligned}
& X_{\mathrm{r}}^{\prime}=(\mathrm{E}, \mathrm{J}, \mathrm{G}, \mathrm{H}) \\
& X_{\mathrm{r}}^{\mathrm{var}}=(\mathrm{C}, \mathrm{D}) \\
& X_{\mathrm{u}}=(\mathrm{A}, \mathrm{B}, \mathrm{F})
\end{aligned}
$$

Minimize: Mass of the customized frame

Subject to: von Mises stress in the member $\leqslant$ $82.7 \times 10^{6} \mathrm{~Pa}$

$f^{61}$

$72^{\circ} \leqslant \mathrm{A} \leqslant 75^{\circ}, 70^{\circ} \leqslant \mathrm{B} \leqslant 74^{\circ}$

$\mathrm{HT}=\left\{\mathrm{HTM}_{\mathrm{S}}, \mathrm{HTM}_{\mathrm{M}}, \mathrm{HTM}_{\mathrm{L}}\right\}$

$f_{\mathrm{var}}^{\wp}=\left\{f_{1}^{\wp}, f_{3}^{\wp}\right\}$

$0.9 \times f_{1}^{\wp} \leqslant C \leqslant 1.1 \times f_{1}^{\wp}$ with $C=f_{1}^{\wp}$

$0.9 \times f_{3}^{\wp} \leqslant D \leqslant 1.1 \times f_{3}^{\wp}$ with $D=f_{3}^{\wp}$
In this case $C$ and $D$ are variables associated with parameters that the user selected to be flexible $\left(X_{\mathrm{r}}^{\mathrm{var}}\right)$. These parameters are now allowed to vary within a range of +10 per cent of their initial value. The set of equations, $f^{\wp}$ that relates user specified parameters with $X_{\mathrm{r}}$ has been divided into $f^{\text {}}$ (relating non-flexible user inputs with product variety parameters) and $f_{\text {var }}^{\wp}$ (relating user inputs specified as flexible with product variety parameters). The second iteration returned a feasible configuration with the altered values of: (a) $\mathrm{HS}=45 \mathrm{~cm}$ and (b) $\mathrm{AR}=56.76 \mathrm{~cm}$. This small change in parameters resulted in a change in von Mises stress by $13.8 \times 10^{6} \mathrm{~Pa}$. The angles A and F play a significant role in the resulting stresses and the maximum von Mises stress is on the joint between lower tube and head tube.

Table 3 MC CAD/FEA template for family of bicycle frames

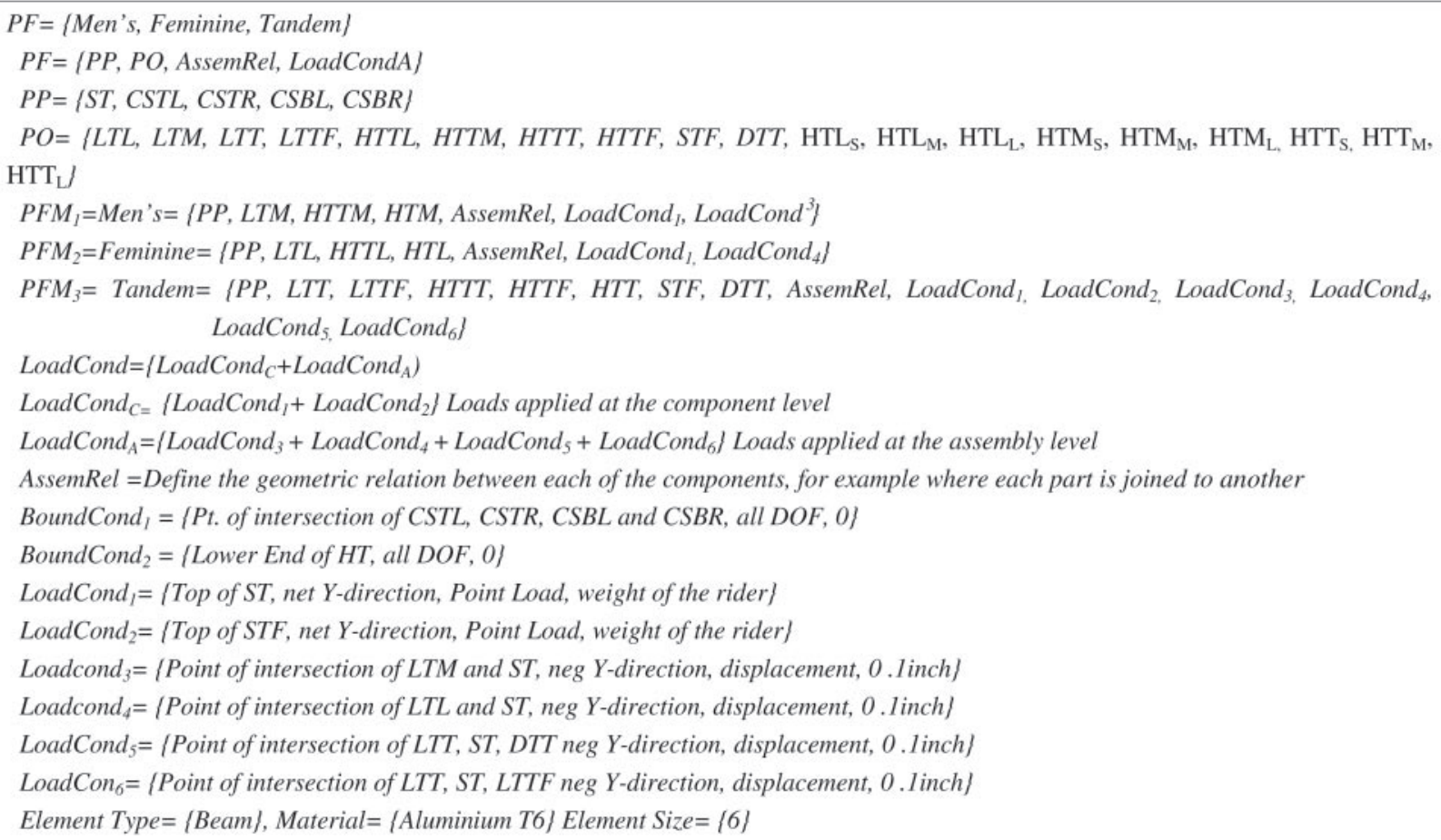
(1) Seat Tube ST
(3) Chain Stay Top Right
CSTR
(5) Chain Stay Bottom Right CSBR
(2) Chain Stay Top Left CSTL
(4) Chain Stay Bottom Left CSBL

Product Optional Components (PO)

(1) Lower Tube Ladies LTL

(2) Lower Tube Men's $\quad$ LTM

(3) Lower Tube Tandem LTT

(4) Lower Tube Tandem Front LTTF

(5) Horizontal Top Tube Ladies HTTL

(6) Horizontal Top Tube Men's HTTM

(7) Horizontal Top Tube Tandem HTTT

(8) Horizontal Top Tandem Front HTTF

(9) Seat Tube Front

(10) Diagonal Tube Tandem

(11) Head Tube Ladies -Small

(12) Head Tube Ladies -Medium

(13) Head Tube Ladies -Large

(14) Head Tube Men's- Small

\begin{tabular}{lll} 
STF & (16) Head Tube Men's- Large & HTM $_{L}$ \\
DTT & (17) Head Tube Tandem-Small & HTT $_{S}$ \\
HTL $_{S}$ & (18) Head Tube Tandem-Medium & HTT $_{M}$ \\
HTL $_{M}$ & (19) Head Tube Tandem- Large & HTT $_{L}$ \\
HTL $_{L}$ & & \\
HTM $_{S}$ & & \\
\hline
\end{tabular}




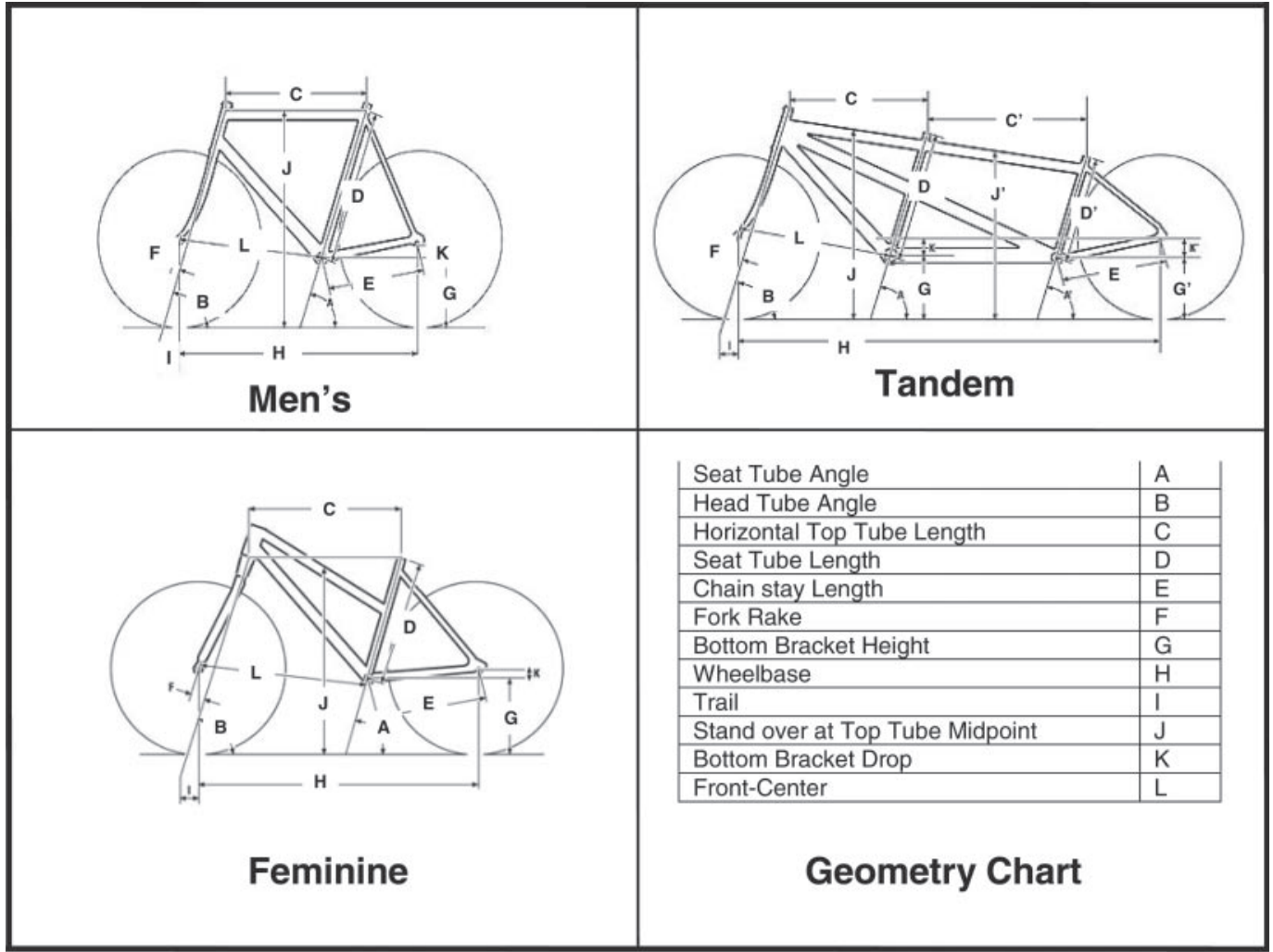

Fig. 4 Geometry chart for men's, tandem, and women's models

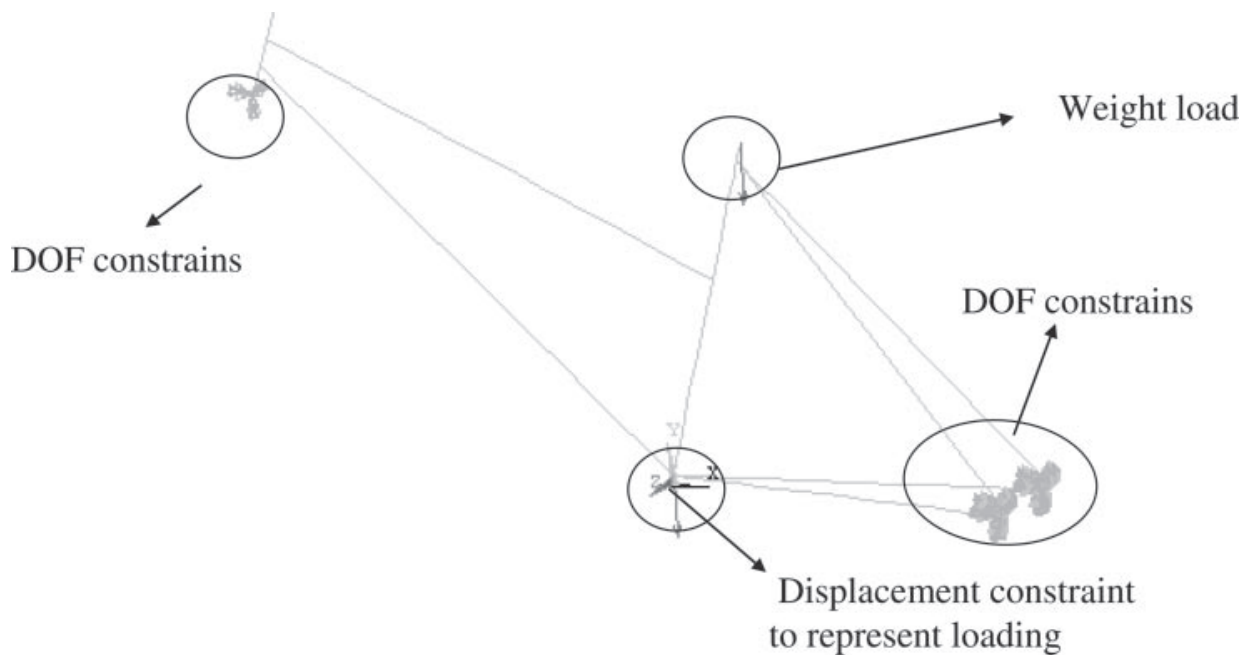

Fig. 5 Finite element model showing loads and boundary conditions

\section{CONCLUSION}

As more and more companies are embracing the PF approach for product development, the design tools and methodologies need to be altered to leverage the product family concept. In the current paper, an internet-based framework was presented to better integrate customers in the design of mass customized products.

The CAD/FEA template presented in the paper takes advantage of the commonality of the $\mathrm{PF}$ members and helps to automate the geometry generation and analysis of different configurations. The template can be applied to automate the design process of modular and scalable PF, to support customization. The optimization of the customized product is a two-round iteration process: if the first round of optimization leads to an infeasible configuration, the customer is prompted to select those parameters that may be altered. The second round of optimization includes these parameters also as design variables. The parameters are allowed to 


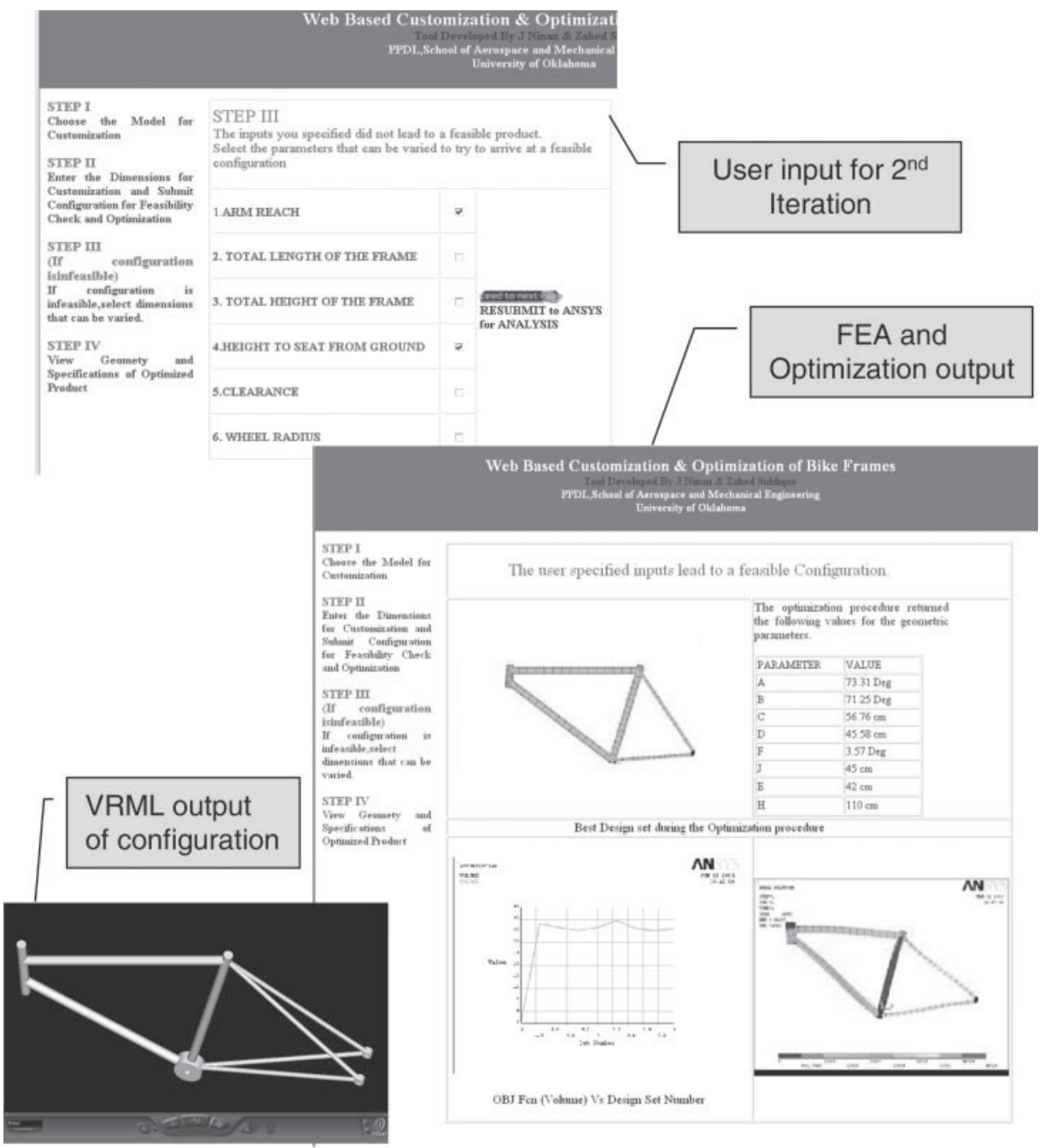

Fig. 6 CAD model, FEA results, and parameters of optimized bicycle frame obtained after second iteration

vary within a range of \pm 10 per cent of the original value. If the second optimization run does not produce any possible configuration either, then the design process is not continued and the customer is just prompted about the infeasible product dimensions. The design iteration needs to be extended until a feasible configuration can be arrived at.

The optimization formulation presented is applicable only to scale-based product architecture with continuous design variables so that gradient-based optimization tools can be used to optimize the user-specified design. In the case of products with discrete variables, even though the same framework can be used, the optimization procedure is time consuming and there is therefore a higher response time to customer requests.

At present, no manufacturing parameters are considered in the optimization process. Since a 3D model of the customized product is generated by the framework, design for manufacturing and other concurrent engineering tools, which use 3D CAD models to perform manufacturing analysis, can be integrated with the framework.

\section{REFERENCES}

1 Gilmore, J. H. and Pine, B. J. I. The four faces of mass customization. Harvard Bus. Rev., 1997, 75, 91-101.

2 Duray, R. and Milligan, G. W. Improving customer satisfaction through mass customization. Quality Progr., 1999, 32(8), 60-66.

3 Wortmann, J. C., Muntslag, D. R., and Timmermanns, P. J. M. Customer driven manufacturing, 1997, (Springer, London).

4 Simpson, T. W., et al. Development of a framework for web-based product platform customization. ASME J. Computing Inf. Sci. Engng, 2003, 3, 119-129.

5 Flores, R., Jensen, C. G., and Shelley, J. A web enabled process for accessing customized parametric designs. In Proceedings of ASME Design engineering technical conferences, 2002, (ASME, Montreal, Quebec, Canada). 
6 Zha, X. F. and Lu, W. F. Knowledge intensive support for product family design. In Proceedings of ASME Design engineering technical conferences - design automation conference, 2002 (ASME, Montreal, Quebec, Canada).

7 Saxon, J. and Beaulieu, C. Combining the power of ANSYS and the WEB - automating stabilizer bar analysis. In AET ANSYS News Letter 4Q2001ARVIN, 2001.

8 Siddique, Z. and Yanjiang, Z. Automatic generation of product family member CAD models supported by a platform using a template approach. In ASME Design engineering technical conferences, 2002 (ASME, Montreal, Quebec, Canada).

9 Siddique, Z. and Boddu, K. An agent based framework to automatically generate $3 \mathrm{D}$ CAD models of customer specified products. In 23rd ASME Computers in engineering conference. 2003, Chicago, USA.

10 Siddique, Z. and Ninan, J. Internet based framework to perform automated FEA on user customized products. In 29th Design Automation Conference, 2003, Chicago, USA.

11 Available online at: http://www.cannondale.com 